\title{
EL SISTEMA FAMILIAR ESPAÑOL. RECORRIDO A TRAVES DEL ULTIMO CUARTO DE SIGLO
}

\section{Angeles Valero}

Universidad Complutense

\section{INTRODUCCION}

A pesar de que Laslett (1972) demostrara que en el Antiguo Régimen la cohabitación de las generaciones no era un fenómeno generalizado en el conjunto de Europa, la instalación de una pareja en un hogar separado forma parte de una tendencia histórica creciente, tendencia, por otra parte, con consecuencias importantes, entre las que destaca el aumento de los hogares de una persona sola entre los grupos de edad más avanzada. El fenómeno se integra dentro de la denominada transición demográfica. Paralelamente, el número de familias con personas no emparentadas ha ido disminuyendo, igualmente, a lo largo del tiempo. Este proceso de nuclearización de la familia moderna llega, en nuestros días, a su máxima expresión en el norte y centro de Europa, en donde las familias plurinucleares apenas alcanzan el 2 por 100.

Aunque la familia constituye una institución universal, las características propias de las distintas sociedades humanas, sus sistemas económicos y su cultura determinan la forma adoptada por esta institución en cada sociedad y momento histórico concreto. Así, el paso de la familia tradicional (preindustrial) a la familia postmoderna ha supuesto un proceso de diferenciación funcional que ha sustituido toda una serie de funciones sociales propias de ese modelo familiar, obligándola a especializarse, para el cumplimiento de dos que le son esenciales: la socialización de los hijos y la estabilidad psíquica y afectiva 
de los adultos, además de la nueva de unidad de consumo. De este cambio se han derivado otros que afectan tanto a la composición interna de la familia, paso de la familia supuestamente extensa a la familia estrictamente nuclear, como a los que modifican su estructura jerárquica y el tipo de relaciones entre sus componentes, transformación de la familia de corte "patriarcal» en familia "simétrica». Es decir, ahora y siempre, la institución familiar está y ha estado inmersa en un constante proceso de transformación paralelo al de la sociedad en que se inscribe, de forma que no se pueden explicar los cambios que se producen en ésta sin tener en cuenta los acaecidos en la sociedad (Le Bras y Bonvalet, 1988: 12).

Básicamente, los factores que han producido, en el último cuarto de siglo, cambios en la estructura familiar en los países occidentales han sido de carácter demográfico y socioeconómico. Dentro de los de carácter demográfico cabe destacar el descenso de la fecundidad, por debajo de los niveles de reemplazo, y el alargamiento de la vida media de los individuos; en relación con los segundos, merecen especial mención el retraso en la edad al matrimonio o ausencia del mismo, unido a un significativo aumento en la incidencia del divorcio, incluyendo elevadas tasas de inestabilidad en las uniones consensuales, de las cuales una buena parte no termina en matrimonio (Burch y Matthews, 1987: 496). Consecuencia de ambos factores es el aumento en la proporción de personas viviendo solas en hogares reducidos, de una o dos personas (unipersonales o matrimonios y parejas sin hijos), que, a su vez, ha producido una disminución del tamaño medio del hogar. A esto hay que añadir la práctica desaparición de los parientes conviviendo en el núcleo familiar y, por supuesto, la de las personas que no son familiares. Parece, pues, fuera de toda duda que la fisonomía de la familia actual ha cambiado, incluso, en cuanto a la terminología que la define, la cual se ha enriquecido con nuevas acepciones, tales como cohabitación (en sustitución de concubinato), familias monoparentales u hogares unipersonales.

\section{Distinción semántica}

Un aspecto de suma importancia dentro de este tema es el de la terminología. El concepto de familia, tal y como usualmente se utiliza, suele implicar otro, el de hogar. Ambos conceptos nunca han sido unívocos, aunque se empleen como tal, por lo que es necesario hacer ciertas precisiones al respecto, especialmente ahora que las formas de convivencia de los individuos se distancian cada vez más de las tradicionales.

Desde el punto de vista sociológico, el término familia incluye diversas realidades, aunque generalmente se aplica a las personas con quien uno vive y a las que está unido por lazos de matrimonio o de consanguinidad; el término puede extenderse a los padres, descendientes no directos, así como a los parientes políticos (Wall, 1983: 7). Además, el término «familia» también es 
utilizado para designar un grupo social y una institución. Como grupo social, la familia está constituida por personas de diferente sexo y edad, vinculadas por lazos de sangre, legales o consensuales, y cuyas relaciones se caracterizan por la intimidad, la solidaridad y la duración.

Por su parte, el hogar tiene un significado mucho más restringido y específico, referido a la residencia compartida, la familiaridad e igualmente las actividades compartidas. El hogar se define, pues, como una unidad económica y social constituida por el conjunto de individuos (puede ser uno solo) que conviven habitualmente bajo el mismo techo y ocupan la misma vivienda. Como lo define Roussel, «el hogar es la unidad elemental de solidaridad cotidiana, unidad que se expresa a través de la permanente cohabitación de sus miembros» (Roussel, 1987: 443).

En función de lo anteriormente dicho, creo que es mucho más adecuado y representativo tomar como objeto de estudio el hogar, su estructura y evolución, en vez de la familia, que, desde las posibilidades que nos ofrecen los datos disponibles, resultaría mucho más impreciso y complicado. Para la consecución de estos objetivos se parte de la hipótesis de que existe una estrecha relación entre el tipo de sociedad, la estructura de los hogares y la composición de los núcleos familiares. De forma que podría deducirse la estructura del hogar de una sociedad determinada conociendo alguna de las características de ésta y, viceversa, se podrá lograr un mejor conocimiento de aquél tomando como indicador su estructura y su tipología.

\section{METODOLOGIA}

Los datos utilizados provienen de los proporcionados por la Encuesta Sociodemográfica realizada por el INE en 1991, y cuyo propósito era el de desarrollar, a partir del correspondiente estudio biográfico, el conocimiento y análisis de las características investigadas por los Censos de Población relativas a los individuos, así como a la evolución de sus situaciones familiares, residencia y vivienda, formación y actividad.

En virtud de su propia identidad, es necesario hacer algunas consideraciones metodológicas que ayuden a facilitar su comprensión. En primer lugar, hay que tener en cuenta que no se trata de un censo y que tanto la metodología empleada, "historias de vida" (método biográfico), como su objetivo difieren de los habituales en el procedimiento censal. En segundo lugar, el universo muestral es distinto (solamente toma las personas mayores de diez años), por lo que las cifras relativas a la estructura de edades y estado civil difieren de las reales. Todo ello no disminuye en modo alguno su valor, permitiendo la comparabilidad con los datos censales. 


\section{FORMAS DE CONVIVENCIA}

\section{Estado civil y formas de convivencia}

Del mismo modo que en España se ha completado, hace ya algún tiempo, la transición demográfica, también se aprecia una transición en el sistema familiar. Así, en el transcurso de los últimos treinta años hemos asistido a un cambio bastante profundo de las estructuras familiares y de hogar, que se manifiesta fundamentalmente en la multiplicación del número de hogares y en la reducción del tamaño medio familiar, junto con un paulatino pero incesante incremento de lo que podríamos denominar nuevas formas de convivencia, producto del cambio social experimentado por nuestra sociedad.

El conocimiento de la distribución de la población, según sus formas de convivencia, en relación con su estado civil, es fundamental para el estudio de las estructuras de hogar, ya que permite saber cuál es la situación familiar de la gente, así como el estado civil predominante dentro de cada situación.

Según los datos del censo de 1991, el 45 por 100 de la población es soltera, el 47 por 100 está casada, el 6 por 100 es viuda y el 1 por 100 está separada o divorciada. Los cambios acaecidos respecto al estado civil en el último decenio pueden calificarse como mínimos. La disminución en la proporción de solteros con respecto a 1981 está más ligada a los cambios en la estructura demográfica (menor tamaño de las cohortes más jóvenes debido al drástico descenso de la fecundidad) que a cambios comportamentales de inclinación al matrimonio. Esta misma explicación es válida para los casados, que deben su incremento $(5,3$ por 100$)$ al progresivo envejecimiento de la población, más que al retraso considerable en la edad al contraer matrimonio que se viene experimentando en el último quinquenio. Por su parte, la proporción de personas viudas dentro de la población española permanece casi inalterada, sin que parezca influir el significativo envejecimiento de sus integrantes. El grupo que más se ha incrementado, en términos relativos (85 por 100), es el de los separados y divorciados, a pesar de lo cual sólo representan el 1 por 100 de la población. Las diferencias por sexo se producen siguiendo la tendencia habitual, una mayor proporción de solteros y casados entre los hombres y menor de viudos y divorciados (tabla 1 ).

En relación con las formas de convivencia, se aprecia fácilmente que la inmensa mayoría de la población vive en familia, es decir, en hogares compuestos por personas de la misma familia (tabla 2). Alrededor del 5 por 100 de las personas mayores de diez años viven solas; de éstas, la mayor proporción corresponde a personas viudas y separadas o divorciadas. En este último caso, la diferencia por sexos es muy llamativa, pues mientras el porcentaje de hombres alcanza el 32 por 100, el de mujeres no supera el 12 por 100; ello es debido a que, ante la ruptura matrimonial, es casi siempre la mujer la que se hace cargo de los hijos, en tanto que el hombre pasa a vivir solo. El número de personas que viven con otras que no sean familiares es realmente exiguo, no lle- 


\section{TABLA 1}

Población según estado civil, 1991

\begin{tabular}{|c|c|c|c|c|}
\hline Estado civil & $\begin{array}{l}\text { Población } \\
\text { sociodem. }\end{array}$ & $\%$ & $\begin{array}{c}\text { Población } \\
\text { censo }\end{array}$ & $\%$ \\
\hline Total. & 34.161 .300 & 100,0 & 38.872 .279 & 100,0 \\
\hline 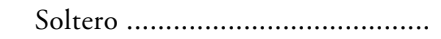 & 13.066 .200 & 38,2 & 17.401 .821 & 44,7 \\
\hline 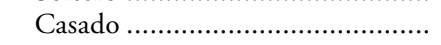 & 18.251 .872 & 53,4 & 18.367 .858 & 47,2 \\
\hline 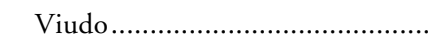 & 2.349 .105 & 6,8 & 2.384 .681 & 6,1 \\
\hline 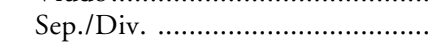 & 485.122 & 1,4 & 447.173 & 1,1 \\
\hline 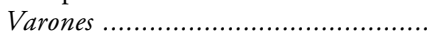 & 16.656 .797 & 100,0 & 19.036 .437 & 100,0 \\
\hline Soltero & 6.936 .442 & 41,6 & 9.123 .511 & 47,9 \\
\hline 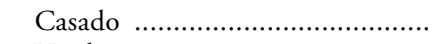 & 9.169 .975 & 55,0 & 9.172 .140 & 48,1 \\
\hline Viudo & 392.959 & 2,3 & 433.361 & 2,2 \\
\hline 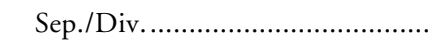 & 157.421 & 0,9 & 175.790 & 0,9 \\
\hline 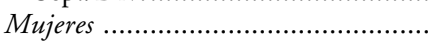 & 17.504 .502 & 100,0 & 19.835 .842 & 100,0 \\
\hline 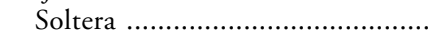 & 6.129 .758 & 35,0 & 8.278 .310 & 41,7 \\
\hline 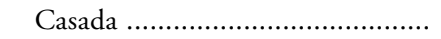 & 9.081 .897 & 51,8 & 9.195 .719 & 46,3 \\
\hline 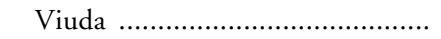 & 1.965 .146 & 11,2 & 1.951 .320 & 9,8 \\
\hline 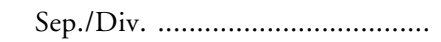 & 327.701 & 1,8 & 271.383 & 1,3 \\
\hline
\end{tabular}

Fuentes: Censo de Población, 1991, INE; Encuesta Sociodemográfica, INE, 1991, y elaboración propia.

gando al 0,5 por 100 de la población en su conjunto; no obstante, esta cifra se eleva algo más, hasta el 2 por 100, cuando se trata de los divorciados y separados, lo cual parece indicar que están viviendo en pareja. En este sentido, conviene reflexionar sobre el hecho de que la forma de convivencia predominante en la sociedad española es la familiar, pese al incremento de otras formas de convivencia denominadas alternativas. No parece que el cambio y la modernización indudable que ha experimentado esta sociedad, mucho más rápido que el de las de nuestro entorno, tenga su correlato en el cambio de la forma de convivencia y, por consiguiente, en la consideración de la familia como unidad de convivencia.

\section{ESTRUCTURA DEL HOGAR}

El modelo teórico que se utiliza como referencia para delimitar las transformaciones demográficas familiares es el de ciclo de vida de la familia, que, aun planteando ciertos problemas metodológicos, se revela como el más idóneo y fácil de utilizar para describir los cambios experimentados por la familia en las últimas décadas. Este modelo fue el primer intento sistemático de crear un modelo teórico que incluyera las diferentes etapas por las que puede pasar cualquier familia. En su representación más común, el ciclo de vida se entien- 
de como una secuencia de eventos: matrimonio (parte del esquema tradicional de que la familia se inicia con el matrimonio y éste, y no otro - cohabitación-, supone la primera etapa del ciclo), primer nacimiento, último nacimiento, salida del hogar del primer hijo, salida del hogar del último hijo y muerte de alguno de los cónyuges. La pertinencia del modelo de ciclo de vida, entre otros factores, se ve afectada por la movilidad matrimonial. La ruptura del matrimonio significa que uno de los cónyuges deja la familia y, si ésta tiene hijos, alguno de los esposos, o ambos, se quedan con alguno de ellos, lo cual supone la creación de dos hogares (familias) a partir de uno solo (Conseil d'Europe, 1990; Ryder, 1985: 211-212). Por tanto, este esquema, aun siendo de utilidad, plantea un cierto número de problemas metodológicos debido a que los patrones actuales de los hogares y de las formas familiares son extremadamente diversos y no pueden ser fácilmente constreñidos a una tipología estándar; no obstante, es el más idóneo y fácil de utilizar para describir las transformaciones que ha experimentado la familia en las últimas décadas (Grebenik, Höhn, Máckensen, 1989).

En el proceso que lleva a una sociedad de la industrialización a la postindustrialización se opera una transformación en la composición del hogar que produce como resultado la reducción del número de miembros del mismo hasta quedar delimitado por el matrimonio y los hijos, frente a la situación anterior en la que el hogar estaba, teóricamente, compuesto por varias generaciones, otros parientes e incluso por miembros no emparentados. La dimensión sociodemográfica del hogar sufre, por consiguiente, unas modificaciones que se manifiestan, en primer lugar, en su tamaño y composición, y en otros aspectos tales como la elección del cónyuge, la duración de la vida familiar, la estructura de las relaciones internas, la frecuencia de la ruptura matrimonial, la función económica, etc. Al mismo tiempo que las biografías individuales de los integrantes dentro de cada familia se van modificando profundamente desde la perspectiva de la familia tradicional, como a continuación se pondrá de manifiesto.

Uno de los rasgos más relevantes del sistema familiar español es su universalización, o sea, que la mayor parte de la población está integrada en la estructura familiar y de hogar, lo cual significa que, a pesar del cambio social y de forma de vida, es muy escasa la población que vive en instituciones o bajo formas diferentes a las de hogar. Por tanto, en la actualidad, y a pesar de las predicciones de hace algún tiempo, no parece que pueda deducirse la desaparición más o menos próxima de las formas de convivencia dentro del hogar y en el seno de la familia, sino más bien todo lo contrario. Ahora bien, lo que sí se está produciendo es un cambio y una complejización de las formas de convivencia y, como consecuencia, de la estructura del hogar. En este aspecto, los rasgos más destacables son la multiplicación de los hogares, la nuclearización de los mismos y la reducción de su tamaño medio.

La población española se ha incrementado un 15 por 100 entre 1970 y 1991, en tanto que el número de hogares lo ha hecho a un ritmo muy supe- 
rior, 25 por 100, en el mismo período. Las causas que han influido en esta transformación son, entre otras, la mejora del nivel de vida, el envejecimiento demográfico y la aparición de formas alternativas de convivencia. La combinación de estos factores se traduce en una mayor nuclearización de los hogares y en la disminución de su tamaño medio. Al mismo tiempo, se acentúa la tendencia en todas las situaciones, tanto maritales como individuales, de formar a partir de un determinado momento un hogar propio, en vez de vivir en hogares extensos. Así, pues, la mayor parte de los cambios mencionados han conducido al aumento de los hogares de unas determinadas características cuya magnitud vamos a constatar en las páginas siguientes.

El total de hogares españoles ascendía a 11.836 .320 en 1991, y se había incrementado, como ya se ha dicho, más de un 25 por 100 con respecto a 1970. Este incremento no se produjo uniformemente en todos los tipos de hogar; muy al contrario, la evolución ha sido muy variada, como lo demuestran los datos que a continuación se presentan (tabla 2).

TABLA 2

Incremento proporcional del número de hogares, 1970-1991

\begin{tabular}{|c|c|}
\hline Tipo de hogar & Incremento 1970-1991 (\%) \\
\hline Hogares sin núcleo (unipersonales) & 140,1 \\
\hline 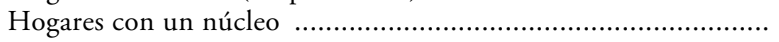 & 37,1 \\
\hline 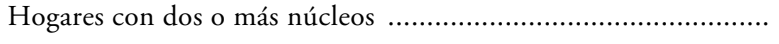 & $-29,9^{*}$ \\
\hline TOTAL DE HOGARES ................. & 25,2 \\
\hline
\end{tabular}

* Datos referidos a 1970 y 1981.

Fuentes: Censo de Población, 1970, 1981, INE; Encuesta Sociodemográfica, INE, 1991, y elaboración propia.

De todos los tipos de hogar, el que más ha aumentado ha sido el de hogares unipersonales, fundamentalmente por el incremento de las personas mayores que viven solas, seguido de los hogares con un solo núcleo, sin duda predominantes, en consonancia con la tendencia de las actuales formas de convivencia. Dentro de la denominación de hogar mononuclear hay que diferenciar diversas clases de unidad familiar: la formada por el matrimonio y los hijos, la constituida por matrimonios solos y la monoparental, madre o padre con sus hijos. Aunque la primera es la que predomina, las otras dos comienzan a alcanzar cifras dignas de consideración. A este respecto, los datos del censo de 1981 son reveladores al mostrar que el hogar mononuclear no constituye un bloque monolítico, sino que más bien es un mosaico de dibujo cambiante. Evidentemente, dentro de este tipo de hogar la estructura que prevalece es la del matri- 
monio con sus hijos solteros, que suponía el 58 por 100 del total de hogares, aunque también había un 18 por 100 de hogares compuestos por matrimonios solos y un 6 por 100 constituidos por madre sola con hijos solteros, además de un 1 por 100 de hogares formados por un padre solo con sus hijos. También se constataba la pervivencia del hogar extenso, que hacía acto de presencia en el 12 por 100 de los hogares nucleares y que está conformado, prácticamente en la totalidad de las ocasiones, por personas relacionadas con el núcleo principal, pero que no son del servicio doméstico. Es decir, que se advierte una diversificación importante dentro de lo que tradicionalmente se denomina hogar nuclear, que sugiere la existencia, y probablemente el incremento, de nuevas estructuras de hogar y de familia (no se cuenta aún con datos para 1991).

En cambio, el retroceso experimentado por los hogares plurinucleares puede calificarse de espectacular y pone de manifiesto las preferencias de la gente hacia una privacidad cada vez mayor (tabla 3).

\section{TABLA 3}

Evolución de los hogares y de su tamaño medio, 1970-1991

\begin{tabular}{|c|c|c|c|c|c|}
\hline & Años & $\begin{array}{c}\text { Hogares } \\
\text { unipersonales }\end{array}$ & $\begin{array}{c}\text { Hogares } \\
\text { mononucleares }\end{array}$ & $\begin{array}{c}\text { Hogares } \\
\text { polinucleares }\end{array}$ & $\begin{array}{l}\text { Tamaño } \\
\text { del hogar }\end{array}$ \\
\hline 1970 & $\ldots .$. & 7,5 & 83,6 & 5,8 & 3,9 \\
\hline 1981 & 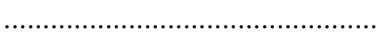 & 10,2 & 83,1 & 3,4 & 3,5 \\
\hline 1991 & 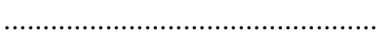 & 13,4 & 85,7 & - & 3,3 \\
\hline
\end{tabular}

Fuentes: Censo de Población, 1970, 1981, INE; Encuesta Sociodemográfica, INE, 1991, y elaboración propia.

En cuanto al tamaño medio del hogar, aunque se aprecia una disminución evidente, ésta no es de la magnitud que cabría esperar, en comparación con otros países de nuestro entorno, especialmente si atendemos al exponencial crecimiento de los hogares unipersonales. Esta aparente paradoja puede obedecer a la permanencia de una buena parte de los jóvenes adultos en el hogar paterno, frenando, en cierta medida, el número de hogares formados por matrimonios solos.

Otra forma de contemplar el tamaño del hogar es analizándolo en relación a la proporción de hogares que hay en cada categoría de tamaño. En este aspecto se evidencia una clara concentración de los valores en torno a aquellos hogares con menos de cuatro miembros. En este cambio han intervenido dos elementos de capital importancia: en primer lugar, el drástico descenso de la fecundidad experimentado en los últimos veinte años (especialmente intenso desde 1980) y, en segundo lugar, la casi desaparición de los hogares plurinucleares y la reducción de los extensos, en los que lo más frecuente es la presen- 
cia de una tercera persona, normalmente anciana, además del núcleo familiar. Los hogares unipersonales son los que han experimentado un mayor incremento, superior al 3 por 100, seguidos de los compuestos por cuatro personas, que han aumentado alrededor de un 1 por 100, y por los hogares compuestos por dos y tres personas, con un incremento inferior al 1 por 100, en ambos casos. Se confirma, una vez más, que el prototipo del hogar español es el compuesto por cuatro personas (padre, madre y dos hijos), seguido por el integrado por dos miembros (matrimonio sin hijos, bien en su primera etapa del ciclo familiar o en la de «nido vacío»). Aún es considerable el peso de los hogares con cinco y más componentes, que suponían, en 1991, el 20 por 100 del total, no obstante haber disminuido un 6 por 100 a lo largo del decenio considerado (tabla 4).

\section{TABLA 4}

Número de personas integrantes del hogar, 1970-1991

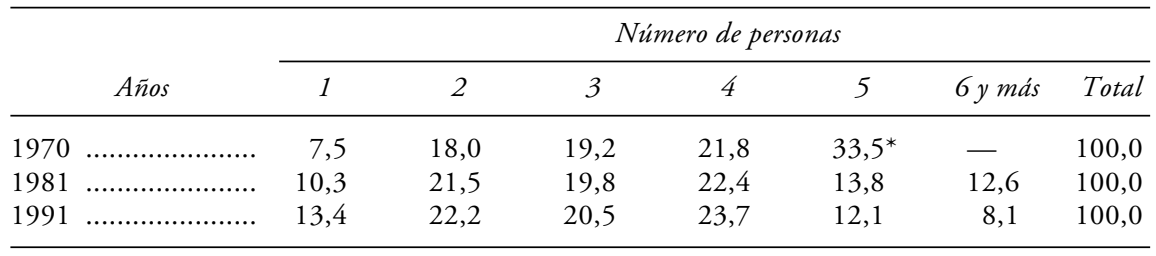

* Incluye cinco y más personas.

FuEnTES: Censo de Población, 1981, INE; Encuesta Sociodemográfica, INE, 1991, y elaboración propia.

\section{ESTRUCTURAS DE HOGAR Y DIVERSIDAD REGIONAL}

La división autonómica supone para nuestros fines un nivel de desagregación suficiente para resaltar los principales rasgos de la estructura de hogar y familia, haciendo posible el establecer comparaciones regionales.

\section{Tamaño y estructura del hogar}

Dentro del cambio estructural experimentado en el sistema familiar, la reducción del tamaño medio del hogar ha sido, sin duda, un fenómeno muy significativo. Dicha reducción se ha producido en todas y cada una de las regiones en el transcurso de los últimos treinta años, pero, eso sí, manteniendo la pauta de tamaño a través del tiempo. Así, Andalucía, Canarias, Cantabria, Galicia y Navarra son las regiones que, a pesar de haber experimentado una 
sensible reducción del tamaño medio del hogar (en ningún caso se alcanzan cuatro personas), se sitúan por encima de la media, en 1991.

En relación con la estructura del hogar, las diferencias regionales son muy significativas y reveladoras de los cambios demográficos y sociales que cada Comunidad Autónoma ha experimentado en el último decenio (tabla 5). Como se ha mencionado anteriormente, el rasgo más llamativo es el incremento de los hogares unipersonales en todas las Comunidades, con un claro incremento en Aragón, Baleares, Castilla y León y La Rioja, regiones todas ellas con un importante grado de envejecimiento y, por tanto, de personas mayores que permanecen solas en sus hogares. Los hogares nucleares (se incluyen de un solo núcleo y de dos o más núcleos debido a que la Encuesta Sociodemográfica no permite su desagregación a este nivel) han experimentado igualmente un extraordinario incremento en todas las regiones, y más especialmente en aquellas zonas en donde tradicionalmente predominaba la familia múltiple y que, debido a la transformación social, ha devenido en familias estrictamente nucleares. Por el contrario, los hogares extensos experimentan una fuerte regresión propia de una sociedad postindustrial.

\section{TABLA 5}

Tipología de los hogares por Comunidades Autónomas, 1981-1991

\begin{tabular}{|c|c|c|c|c|c|c|}
\hline \multirow[b]{2}{*}{ Comunidad } & \multicolumn{2}{|c|}{ H. unipersonales } & \multicolumn{2}{|c|}{$\begin{array}{c}\text { H. mononucleares } \\
\text { y polinucleares }\end{array}$} & \multicolumn{2}{|c|}{ H. extensos } \\
\hline & 1981 & 1991 & 1981 & 1991 & 1981 & 1991 \\
\hline Andalucía & 9,3 & 11,8 & 76,0 & 87,6 & 12,2 & 1,5 \\
\hline Aragón & 11,8 & 16,5 & 73,1 & 82,7 & 11,5 & 1,1 \\
\hline 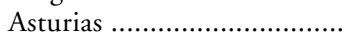 & 10,5 & 14,0 & 72,2 & 85,2 & 12,4 & 1,4 \\
\hline 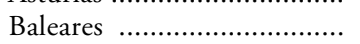 & 13,9 & 17,1 & 72,5 & 81,9 & 10,0 & 1,4 \\
\hline 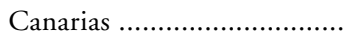 & 8,8 & 10,7 & 78,1 & 88,3 & 10,1 & 1,6 \\
\hline 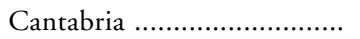 & 10,1 & 13,1 & 74,1 & 85,6 & 12,2 & 1,9 \\
\hline Castilla y León ......................... & 12,7 & 16,2 & 73,6 & 83,1 & 10,1 & 1,0 \\
\hline Castilla-La Mancha .............. & 11,3 & 14,2 & 76,3 & 85,5 & 8,5 & 0,5 \\
\hline 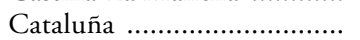 & 9,9 & 13,3 & 73,8 & 85,7 & 10,2 & 1,5 \\
\hline Extremadura ......................... & 10,1 & 14,4 & 73,7 & 84,9 & 11,8 & 1,0 \\
\hline 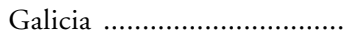 & 10,1 & 13,1 & 69,4 & 85,8 & 13,5 & 1,7 \\
\hline 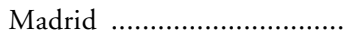 & 10,4 & 13,2 & 74,1 & 85,0 & 9,2 & 2,5 \\
\hline 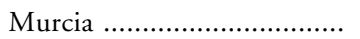 & 8,9 & 11,4 & 78,6 & 88,2 & 9,8 & 0,5 \\
\hline 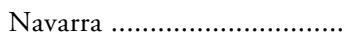 & 10,2 & 13,8 & 74,7 & 85,1 & 11,3 & 1,7 \\
\hline 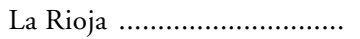 & 11,4 & 15,6 & 75,8 & 83,7 & 9,8 & 1,1 \\
\hline C. Valenciana ......................... & 10,3 & 13,8 & 76,6 & 85,6 & 10,5 & 1,0 \\
\hline 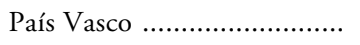 & 8,0 & 12,4 & 76,1 & 86,5 & 11,9 & 1,6 \\
\hline TOTAL NACIONAL ........... & 10,3 & 13,4 & 74,5 & 85,7 & 11,8 & 1,8 \\
\hline
\end{tabular}

Fuentes: Censo de Población, 1981, INE; Encuesta Sociodemográfica, INE, 1991, y elaboración propia. 


\section{Estructura del hogar y edad de sus componentes}

Otro aspecto importante a considerar en el tema que nos ocupa es el de la composición demográfica de los componentes del hogar. Efectivamente, el ciclo de vida del hogar, así como su estructura, dependen de la etapa del ciclo de vida de sus integrantes, y, por tanto, el hecho de tener menores y/o personas ancianas afecta directamente las características estructurales del hogar, por no hablar del tipo de relaciones intrafamiliares.

Al igual que ha ocurrido con la población, en el transcurso de los últimos treinta años se ha producido un clarísimo envejecimiento de los hogares españoles en su conjunto. En 1991, el número de hogares con niños menores de dieciséis años había sufrido un descenso del 9 por 100 con respecto a 1981, en tanto que el de aquellos con alguna persona de más de sesenta y cinco años se incrementó en un 29 por 100. De los datos presentados por la Encuesta Sociodemográfica se desprende que, en 1991, el 60 por 100 de las familias no convive con ningún niño o adolescente. Este fenómeno parece ser el resultado de diversos fenómenos concomitantes: en primer lugar, el importante descenso de la natalidad, que hace que el número de hijos por familia haya descendido drásticamente; en segundo, el retraso por parte de muchas parejas jóvenes a la hora de tener el primer hijo, tal y como lo demuestra el hecho de que apenas el 12 por 100 de los hogares tuvieran un miembro menor de cuatro años; $y$, en tercero, el aumento de los hogares formados por matrimonios solos de edad madura, en su fase de contracción del hogar (gráfico 1).

\section{GRAFICO 1}

Hogares con personas menores de dieciséis años por CC.AA.

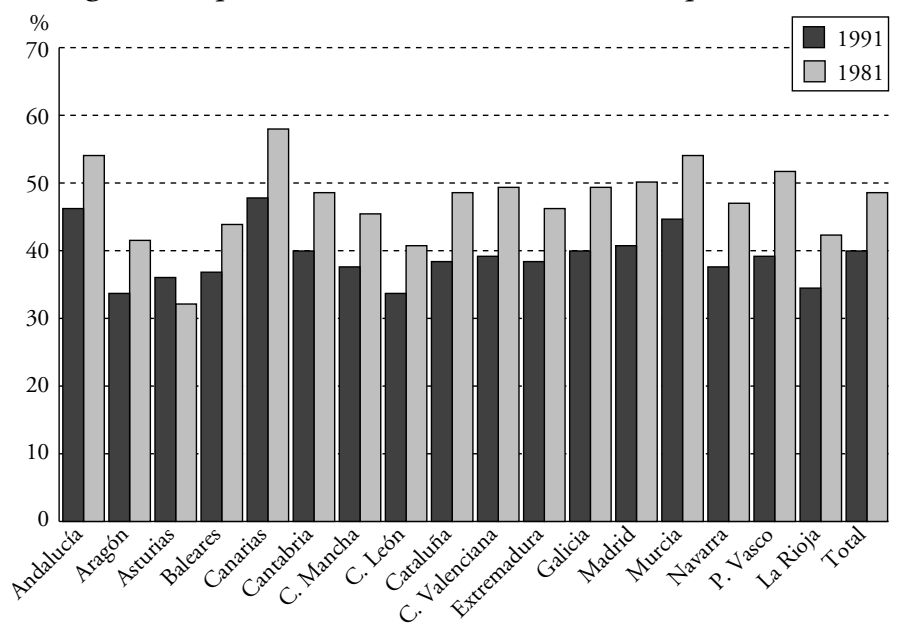

COMUNIDADES AUTONOMAS 
A nivel autonómico se aprecia que la pauta general es la misma, eso sí, conservando reminiscencias de pautas anteriores; de hecho, en todas las Comunidades, con la excepción de Asturias, ha disminuido la proporción de menores en los hogares con respecto a 1981. Aun así, Andalucía, Canarias y Murcia, a pesar del descenso, mantenían, todavía en 1991, valores superiores a la media en cuanto al número de hogares con menores de dieciséis años.

La conjunción de ciclo de vida personal y la de ciclo de vida familiar se refleja muy claramente cuando se estudia el número de personas mayores de sesenta y cinco años conviviendo en familia. En el caso de una población en claro proceso de envejecimiento como es la española, no es sorprendente el hecho de que, en 1991, el 34 por 100 de los hogares integrasen a alguna persona con más de sesenta y cinco años y, asimismo, que en el 10 por 100 de los hogares conviviera un anciano de más de ochenta años. A su vez, se debe resaltar el hecho de que exista un buen número de hogares formados únicamente por personas mayores; ésta era la situación del 16 por 100 de los hogares cuyos componentes tienen todos más de sesenta y cinco años (gráfico 2).

\section{GRAFICO 2}

Hogares con personas mayores de sesenta y cinco años por CC.AA.

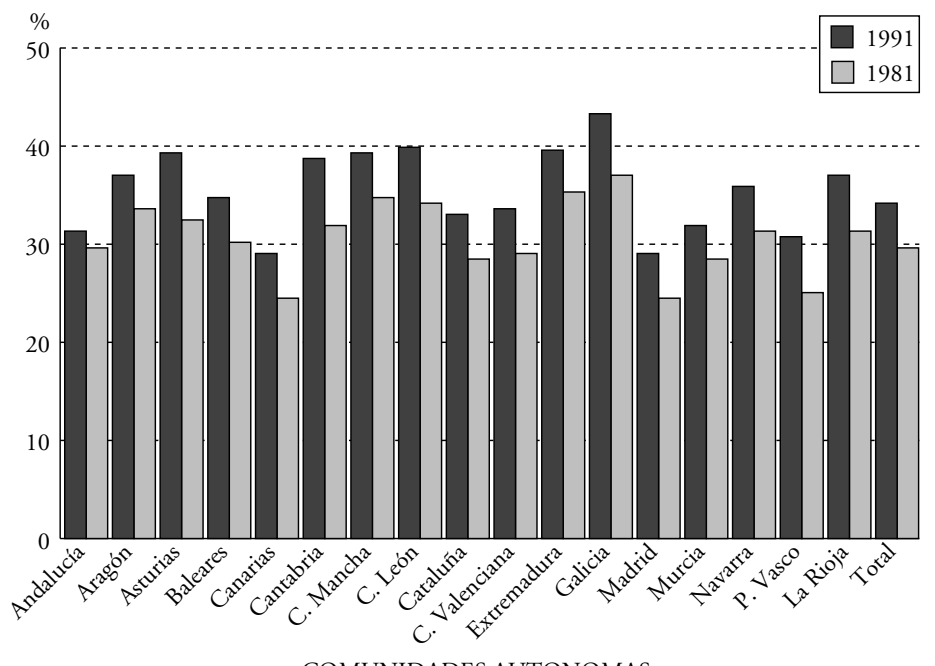

COMUNIDADES AUTONOMAS

En este caso, las Comunidades más envejecidas demográficamente, o aquellas con mayores desequilibrios como consecuencia de la emigración, son las que progresivamente han incrementado el número de hogares con personas mayores, como es el caso de Aragón, Asturias, Cantabria, Castilla-La Mancha, Castilla y León, Extremadura y Galicia. 
Asimismo, es igualmente importante el hecho de que el 12 por 100 de los hogares españoles sean intergeneracionales, es decir, que en ellos convivan al menos tres generaciones; manteniéndose de este modo la relación de convivencia entre los jóvenes, los adultos y los ancianos, con toda la riqueza social y afectiva que ello supone.

En resumen, de los datos presentados se desprende la enorme transformación estructural que está sufriendo la familia española; así, observamos que en tanto que el 18,5 de los hogares cuenta entre sus componentes con un niño menor de seis años, el 30 por 100 tiene entre sus miembros, al menos, una persona de entre sesenta y cinco y setenta y nueve años, lo cual da idea del proceso de envejecimiento de nuestra sociedad tanto a nivel individual como familiar.

\section{CONCLUSIONES}

A la vista de lo anteriormente expuesto, creo que no es descabellado concluir que el sistema familiar y de estructura de hogar español mantiene unos rasgos y características propios que le hacen diferente, incluso, de los del entorno mediterráneo, exceptuando a Italia.

Los rasgos fundamentales que caracterizan el sistema familiar en Europa, en términos generales, son: aumento constante del número de hogares, aumento de las familias monoparentales, aumento de los jóvenes que viven solos y mayor número de personas mayores viviendo solas en sus propios hogares. Todo ello da como resultado un cada vez mayor número de hogares, con un tamaño incesantemente más reducido y un menor peso relativo de los hogares conyugales (marido, mujer e hijos). Por el contrario, los rasgos definitorios de nuestro sistema familiar se centran en el aumento constante del número de hogares, debido, básicamente, a la multiplicación del número de ancianos que viven solos (hogares unipersonales) y al considerable incremento de los núcleos familiares (padre, madre e hijos), así como al de los hogares intergeneracionales, frente a una muy baja proporción de hogares monoparentales o de cohabitantes e incluso de jóvenes adultos viviendo solos. Esta combinación de factores da como resultado que el tamaño medio del hogar sea algo más elevado que el de los otros países, a pesar del prolongado descenso de la fecundidad.

Todo ello sin que, al mismo tiempo, deje de advertirse una tendencia a una mayor diversidad de formas de convivencia, de tipos de hogares y de relaciones familiares, que conllevan una mayor complejidad en las relaciones de parentesco y en las vinculaciones entre unos y otros hogares. 


\section{BIBLIOGRAFIA}

Bongants, J. (1987): Family Demography. Methods and their Applications, Clarendon Press, Oxford.

Bourgeois-Pichat, J.; Festy, P. (1990): «19 e rapport sur la situation démographique de la France», Population, $45^{\mathrm{e}}$ année, n. ${ }^{\circ}$ 4-5 (pp. 873-921).

Burch, T.; Matthews, B. J. (1987): "Household Formation in Developed Societies», Population and Development Review, n. ${ }^{\circ}$ 3, September (pp. 485-512).

CONDE, R. (comp.) (1982): Familia y cambio social en España, CIS, Madrid.

CONGRÉS ET COlloques (1990): La famille dans les pays développés: Permanences et changements, INED, Paris.

Conseil De L'Europe (1990): «La structure des ménages en Europe», Etudes Démographiques, n. ${ }^{\circ} 22$, Strasbourg.

- (1993): Recent demographic developments in Europe and North America 1992, Strasbourg.

Del Campo, S. (1991): La nueva familia española, Eudema, Madrid.

Garrido, L., y Gil Calvo, E. (eds.) (1993): Estrategias familiares, Alianza Universidad, Madrid.

Grebenik, E.; Höhn, Ch.; Mackensen, R. (eds.) (1989): Later Phases of the Family Cyle. Demographic Aspects, Clarendon Press, Oxford.

Goldscheider, F. K.; Waite, L. (1991): New Families, no Families?, University California Press, Berkeley.

Hoffmann Nowotny, H. (1987): «The Future of the Family», European Population Conference, IUSSP, Helsinki (pp. 112-198).

IgLesias De Ussel, J. (ed.) (1988): Las familias monoparentales, Instituto de la Mujer, Ministerio de Asuntos Sociales, Madrid.

Laslett, P.; Wall, R. (eds.) (1977): Household and Family in the Past Time, Cambridge University Press, Cambridge.

LE Bras, H.; BonvaleT, C. (1988): Transformation de la famille et habitat, INED, Paris.

Leridon, H. (1990): «Extra-Marital Cohabitation and Fertility», Population Studies, n.o 44 (pp. 469-487).

LeRIDON, H.; VilleneuVe-GoKalp, C. (1988): «Les nouveaux couples: nombre, caractéristiques et attitudes», Population, $43^{\mathrm{e}}$ année, n. ${ }^{\circ} 2$ (pp. 331-367).

Lesthaeghe, R. (1983): «A Century of Demographic Cultural Change in Western Europe: An Exploration of Underlying Dimensions", Population and Development Review, 9, 3 (pp. 411 435).

Lesthaeghe, R.; Meekers, D. (1986): «Value Changes and the Dimensions of Familism in the European Community», European Journal of Population, n.o 2 (pp. 225-268).

Monnier, A. (1990): «La conjoncture démographique: l'Europe et les pays développés d'OutreMer», Population, $45^{\mathrm{e}}$ année, n. ${ }^{\circ} 4-5$ (pp. 924-936).

RALLU, J. L. (1986): "Les effets des nouveaux rythmes familiaux sur la repartition des familles selon le type et la taille», Les familles d'aujourd'hui, Colloque de Genève (17-20 septembre 1984), AIDELF, Paris.

Roussel, L. (1985): «Le cycle de la vie familiale dans la société post-industrielle», International Population Conference, IUSSP, Florence, F. 13. 2.

- (1986): "Evolution récente de la estructure de ménages dans quelques pays industriels», Population, n. ${ }^{\circ} 41$ (pp. 913-934).

- (1987): «Deux décennies de mutations démographiques (1965-1985) dans les pays industrialesés», Population, n. ${ }^{\circ} 3$, mai-juin (p. 443).

- (1989): La famille incertaine, Editions Odile Jacob, Paris.

- (1991a): «Donnés démographiques et structures familiales», L’Année Sociologique, vol. 37 (pp. 123-138).

- (1991b): "Les "futuribles de la famille"», Futuribles, n. ${ }^{\circ} 153$ (pp. 3-22).

- (1992): «La famille en Europe occidental: divergences et convergences», Population, $47^{\mathrm{e}}$ année, n. ${ }^{\circ} 1$ (pp. 133-151). 
- (1993): Fertility and Family, European Population Conference, 23-26 March, Council of Europe.

Roussel, L.; Festy, P. (1979): Recent Trends in Attitudes and Behavior affecting the Family in Council of Europe Member States, Council of Europe, n. ${ }^{\circ} 4$, Strasbourg.

Valero, A. (1990a): «Evolución del hogar y de la estructura familiar en Madrid», Boletín de Demografía Histórica, IX, n.o 1 (pp. 89-121).

- (1990b): "Cambios en la estructura del hogar y de la familia en Madrid», Demografía urbana y regional, $1 .^{\text {as }}$ Jornadas Internacionales, Consejo Superior de Investigaciones Científicas, Instituto de Demografía, Madrid (pp. 45-78).

- (1992): «La prevalencia de la familia nuclear en el sistema familiar español», Revista Internacional de Sociología, n. ${ }^{\circ}$ 3, septiembre-diciembre (pp. 183-210).

- (1993): «La muerte de la familia. ¿Mito o realidad?», en varios, Escritos de Teoría Sociológica, Centro de Investigaciones Sociológicas, Madrid (pp. 1127-1143).

Valero, A.; Pérez Lence, C. (1995): «Nupcialidad, fecundidad y familia. La paradoja del comportamiento de la nupcialidad y la fecundidad en España», Revista Internacional de Sociologia, n. 11 (pp. 89-113).

Wall, R. (ed.) (1983): Family Forms in Historic Europe, Cambridge University Press, Cambridge.

\section{RESUMEN}

El sistema familiar español ha evolucionado de forma muy semejante a lo acontecido en los países de la Europa occidental. Es decir, se constata un aumento constante del número de hogares, un aumento del número de hogares unipersonales y una reducción del tamaño medio familiar. Pero conjuntamente con estos rasgos se dan otros que hablan de forma inequívoca de la peculiaridad de nuestro sistema familiar, a saber: crecimiento constante de los núcleos familiares (padre, madre e hijos) y de los hogares intergeneracionales frente a una todavía débil proporción de hogares monoparentales, de cohabitantes y, por supuesto, de jóvenes adultos viviendo solos.

\section{ABSTRACT}

The development of the family system in Spain has followed much the same pattern as in other countries of Western European: the number of households and single-person homes in increasing, whereas the average family size is shrinking. But at the same time, other factors clearly attests to the peculiarity of the Spanish family system, namely the constant growth of family nuclei (mother, father, children) and of households in which different generations live together, in contrast to a still small proportion of single-parent households, of unmarried couples living together and of young adults living alone. 